\title{
Mujeres migrantes en albergues para jornaleros agrícolas: una aproximación a la vulnerabilidad social
}

\section{Migrant women in agricultural laborer's shelters: an approach to social vulnerability}

\author{
GUILLERMINA CHÁVEZ TORRES \\ Doctora en Ciencias Sociales, Profesora Investigadora de la Facultad de Trabajo Social, \\ Universidad de Colima (México), gctorres@ucol.mx - ORCID ID: 0000-0001-6741-5313
}

Recibido: 30/08/2020

Aceptado: 10/11/2020

\begin{abstract}
Resumen: El estudio tiene como objetivo describir la vulnerabilidad social a la que están expuestas las mujeres migrantes que viven temporalmente en albergues para jornaleros en el corte de caña en el municipio de Cuauhtémoc, Colima, México. Se diseñó un estudio exploratorio que permitiera describir elementos macro y micro objetivos que conforman el espacio temporal de mujeres migrantes a través de la categoría analítica de vulnerabilidad social. Los resultados preliminares del estudio ${ }^{1}$ permitieron identificar que la vulnerabilidad social a la que están expuestas las mujeres estudiadas, se traduce en una pobreza generacional que se ha configurado por un sistema de exclusión que responde a su lugar de origen y el idioma indígena, una limitación al desarrollo de su capital humano por el rezago educativo y una restricción al desarrollo de su capital social por el desplazamiento pendular.
\end{abstract}

Palabras clave: mujeres migrantes, vulnerabilidad social, pobreza, capital humano y social, trabajo social.

\begin{abstract}
The objective of this paper is to describe the social vulnerability that migrant women, temporally living in the cane cutting laborer's shelters of Cuauhtemoc, Colima Mexico, are exposed to. The exploration study was made using the analytical category of social vulnerability. The main results were the identification of macro and micro objective factors that signal to the existence of a limitation to the human capital development due to the educational lag, the generational poverty is configured by an exclusion system starting from the place of origin and the indigenous language, and the pendular displacement that hinders the social capital development.
\end{abstract}

Key words: migrant women, social vulnerability, poverty, human and social capital, social work. 1 Este artículo se deriva del proyecto Vulnerabilidad social de familias jornaleras migrantes en el corte de caña en Colima realizado de septiembre de 2018 a enero de 2020 con
un financiamiento del Programa para el Desarrollo Profesional Docente (PRODEP) en la convocatoria de NPTC (2018). 


\section{INTRODUCCIÓN}

La combinación de los fenómenos de pobreza y migración, crea una condición que demanda el involucramiento en distintos niveles del Trabajo Social, el cual busca incidir favorablemente para promover "el cambio y el desarrollo social, la cohesión social, y el fortalecimiento y la liberación de las personas" (Federación Internacional de Trabajadores Sociales, 2014, párr. 1).

En ese sentido, desde el Trabajo Social se buscó tener una aproximación a la vulnerabilidad social a la que están expuestas las mujeres migrantes de las familias jornaleras que se incorporan en el corte de caña en Colima, México, con el interés que tiene la disciplina del conocer para actuar.

Es necesario resaltar, que cuando se escribe sobre las mujeres migrantes de familias jornaleras, se enfoca la mirada en el grupo de mujeres que permanecen en los albergues, ya sea en calidad de madres, esposas o hijas de jornaleros, que se dedican al trabajo doméstico, que contribuyen a la dinámica cotidiana y que, desafortunadamente, hay poco escrito sobre ellas.

En una panorámica general mexicana, la división por sexo de quienes se dedican a la actividad jornalera, "de acuerdo con la Encuesta Nacional de Ocupación y Empleo (ENOE) para 2017 [...] la población es del 20\% mujeres y el $80 \%$ son hombres jornaleros. Dentro de este grupo la participación de las mujeres en los campo agrícolas ha crecido" (citada en Red Nacional de Jornaleros y Jornaleras Agrícolas, 2019, p. 15).

Mientras que, de acuerdo con la Encuesta Nacional de Ingresos y Gastos de los Hogares 2015, (citada en Red Nacional de Jornaleros y Jornaleras Agrícolas, 2019) hay 5 millones 955 mil 889 personas, como población potencial2 ${ }^{2}$, siendo 1 millón 543 mil 400 jornaleros y jornaleras agrícolas y, el resto, 4 millones 412 mil 489 son integrantes de su familia. Sin embargo, las cifras resultan ser una estimación, ya que el desplazamiento temporal imposibilita tener el registro, pues las personas jornaleras agrícolas cada vez más, suelen viajar con sus familias (Secretaría de Gobernación, 2018). Por lo que es menester, considerar también a quienes integran las familias.

Existen condiciones de las familias mencionadas que han sido presentadas con información de sus problemas prácticos, tales como, la falta de empleo en sus lugares de origen, la inseguridad e irregularidad de sus traslados migratorios o sobre las circunstancias de hacinamiento y la informalidad laboral al que están expuestas en el lugar de destino (Grupo Sinergia, 2011; Olmos, 2016; Red Nacional de Jornaleros y Jornaleras Agrícolas, 2019; Secretaría de Desarrollo Social, 2010).
Particularmente, sobre la configuración social de las familias jornaleras migrantes, hace que "el acceso a derechos como la salud y la vivienda se vean sumamente comprometidos. Además, su necesidad de movilidad constante también tiene efectos negativos sobre la posibilidad de que sus hijas e hijos permanezcan en el sistema educativo" (Secretaría de Gobernación, 2018 , p. 2). La suma de los problemas prácticos referidos ha dado como resultado a lo que se conoce como "círculos viciosos en los que se transmite de generación en generación la pobreza" (Secretaría de Desarrollo Social, 2010, p. 42).

Dentro de la agenda 2030, que se enmarca en los Objetivos de Desarrollo Sostenible, las problemáticas de las familias jornaleras migrantes, inciden en varios objetivos que se desean alcanzar, tales como el fin de la pobreza, la igualdad de género, salud y bienestar, educación de calidad, el hambre cero y la reducción de las desigualdades (Programa de las Naciones Unidas para el Desarrollo, 2015); por lo tanto, la información que se genere para avanzar en conocimiento $y$, que pueda traducirse en acciones de intervención que permitan prosperar en el logro de los objetivos, justifica su pertinencia.

En un acercamiento a los estudios sobre las familias jornaleras migrantes, se identificó una forma oblicua y empañada en la que se han abordado algunos de estos, ya que se ha argumentado que los problemas de vulnerabilidad y exclusión social, están originados "por falta de educación y desconocimiento y por falta de organización social" (Secretaría de Desarrollo Social, 2010, p. 11) por parte de la población, dejando de lado factores como el contexto geográfico e histórico, que no pueden mantenerse al margen.

Asimismo, la realidad de las mujeres migrantes está desdibujada del contexto mencionado, en el sentido de que no existe información o registro de aquellas que no se incorporan en alguna actividad laboral formal o en su caso, que no asisten a la escuela, por lo que no hay acciones de bienestar dirigidas a ellas.

También, se ha expuesto que la migración es un problema, sin embargo, desde una postura de derechos humanos, la migración es una condición natural del ser humano y puede ser concebida como "una estrategia de intervención al permitir al individuo desarrollar y rentabilizar sus aptitudes en el lugar de destino" (Pimienta y Vera, 2015, p. 10). Postura que invita a reflexionar que el enfoque de la mirada debería estar en otros elementos que han condicionado la realidad social de las familias jornaleras migrantes.

La complejidad en la que se enmarca la realidad mencionada demanda al Trabajo Social, buscar mejores herramientas analíticas y novedosas formas de acercamiento, que le

2 Mujeres y hombres de 16 años o más que laboran como jornaleros/as agrícolas, así como los integrantes de su hogar. 
permitan conocer de forma integral la situación e incidir de forma positiva, en la realidad social que configuran las mujeres migrantes de familias jornaleras.

\section{ESTRATEGIA DE ANÁLISIS: VULNERABILIDAD SOCIAL}

Para conocer la compleja realidad que viven las mujeres migrantes de familias jornaleras agrícolas, se concibió una estrategia analítica bajo el paradigma crítico; considerando lo que señaló Mancinas (2017) "no es posible comprender a la realidad social como algo autónomo de la acción humana, de la misma manera que es imposible analizar al sujeto al margen de su contexto histórico" (p. 157), agregaría, ni a su contexto geográfico, pues influyen en el desarrollo y acceso a oportunidades de bienestar social.

Bajo la propuesta de los diferentes niveles de análisis social que planteó Ritzer (2002) se construye un continuum social, al que se accede cuantitativamente y cualitativamente, para este estudio como acercamiento inicial, se eligió acceder a los niveles macro y micro objetivos. El nivel macro objetivo refiere a las estructuras formales como lo son las instituciones sociales que se vinculan con la realidad estudiada y, el nivel micro objetivo, alude al contexto que ha determinado la transmisión generacional de estilos de vida, como lo es la migración temporal para insertarse en actividades laborales agrícolas.

Teniendo como marco, el paradigma crítico y el nivel de análisis macro y micro objetivo, se encuadra a la vulnerabilidad social, que como categoría analítica ha tenido diversas conceptualizaciones, por ejemplo, la que se ha utilizado para estudiar la pobreza y que se relaciona directamente con la situación de las mujeres migrantes estudiadas:

Se relaciona con la posibilidad de captar la forma y las causas por las que diversos grupos sociales están sometidos a eventos y procesos que atentan contra su capacidad de subsistencia, su acceso a mayores niveles de bienestar y el ejercicio de sus derechos ciudadanos (Golovanevsky, 2007, pp. 55-56)

Asimismo, una forma de concebir la vulnerabilidad y su unidad de análisis, es la que señala Ruíz (2012):

Vulnerabilidad se define siempre en relación con algún tipo de amenaza, sean eventos físicos [...] o amenazas antropogénicas [...]

La unidad de análisis (individuos, hogar, grupo social) se define como vulnerable ante una amenaza específica, o es vulnerable a estar en una situación de pérdida, que puede ser salud, del ingreso, de las capacidades básica, etc. (p. 64)

En términos generales, la vulnerabilidad social permite captar la complejidad que debe ser estudiada, "ya que tiene dimensiones analíticas e incluye aspectos de individuos y hogares, así como también características económicas, políticas, culturales y ambientales de la sociedad. Por ello [...] tiene un carácter polisémico y da lugar a múltiples enfoque metodológicos" (Busso, 2002, citado en Golovanevsky, 2007, p. 56).

En suma, la vulnerabilidad social se ha utilizado, para captar "las estrategias que utilizan [grupos] para sobrevivir y en algunos casos salir de la pobreza desde el enfoque de activos y vulnerabilidad" (Golovanevsky, 2007, p. 56). Es así como se tuvo la aproximación a los factores de vulnerabilidad social a la que están expuestas las mujeres migrantes y que las ha lleva a formar parte de la pobreza generacional.

Para el estudio que se presenta, se entiende a la vulnerabilidad social bajo el modelo de Golovanevsky (2007) que refiere al ensamblaje entre los recursos (simbólicos y tangibles) que tiene la población, (acceso/limitación) la estructura de oportunidades y, las instituciones y el tejido de sus relaciones sociales, ejes que se han adaptado a las características de la población estudiada y se describen a continuación:

a. Los recursos: indican los rasgos demográficos, el tipo de vivienda temporal en la que se asienta la población migrante en el lugar de destino y el capital humano que han desarrollado. Recursos en el sentido de "posesión, control o movilización de recursos, tanto materiales como simbólicos, que permiten desempeñarse en la sociedad" (Golovanevsky, 2007, p. 58).

b. Estructura de oportunidades: este eje está determinado por el tipo de empleo al que acceden las familias estudiadas y en consecuencia, a la protección social a la que acceden por el empleo y a la intervención del Estado en forma de política social, como estructura formal de desarrollo o bienestar social y, otros organismos involucrados; en palabras de Golovanevsky (2007) "proviene del mercado, del Estado y de la Sociedad" (p. 58).

$c$. Instituciones y relaciones sociales: este elemento refiere al capital social, los antecedentes familiares y la composición familiar, que permite y ha perpetuado la forma y el estilo de vida de las mujeres migrantes y sus familias, en el sentido de que "las diferentes formas de relación y sociabilidad que las personas desarrollan como modalidades de acción colectiva" (Golovanevsky, 2007, p. 58) y que en la vida cotidiana pueden traducirse en una estructura de apoyo. 
Adicionalmente, para referir a los elementos de vulnerabilidad social se consideró la interseccionalidad partiendo que

consiste en aprehender las relaciones sociales como configuraciones históricas que forman lo que Candace West y Sarah Fentersmaker llaman realizaciones situadas, es decir, contextos en los cuales las interacciones de las categorías de raza, clase y género actualizan dichas categorías y les confieren significados (Viveros, 2016, p. 12).

La realidad de las familias jornaleras migrantes es compleja por el sistema de exclusión social al que están expuestas, la interseccionalidad reconoce que las mujeres, de las mencionadas familias, tienen condiciones especiales que deben identificarse por "los complejos, irreductibles, variados y variables efectos que resultan cuando múltiples ejes de diferencia - económica, política, cultural, psíquica, subjetiva y experiencial- se intersectan en contextos históricos específicos" (Brah, 2013, p. 14).

Dentro de la estructura de la vulnerabilidad social, la interseccionalidad asiente que los "factores como raza, etnia, religión o creencias, salud, estado civil, vivienda, estatus migratorio, edad, discapacidad, orientación sexual, identidad de género, las mujeres pueden tener especiales necesidades y ser más vulnerables a las discriminaciones múltiples" (La Barbera, 2016, pp. 115-116), es decir, existe una realidad particular y diferenciada para las mujeres migrantes de familias jornaleras agrícolas.

\section{ACERCAMIENTO EXPLORATORIO A LA VULNERABILIDAD SOCIAL}

Para tener el primer acercamiento exploratorio a la vulnerabilidad social a la que están expuestas las mujeres estudiadas, se utilizó una encuesta para obtener información de esa parte de la realidad social a la que interesaba llegar. El supuesto de investigación fue que la mayoría de las mujeres migrantes en los albergues, que no se incorporan a una actividad laboral ni asisten a una institución educativa, se encuentran invisibilizadas sumando mayores elementos a su vulnerabilidad social.

Como instrumento, se diseñó un cuestionario compuesto de 59 reactivos, los cuales se organizaron en secciones, a decir, de datos generales, servicios de salud, estructura familiar, datos laborales de proveedoras(es) principales, comunicación, discapacidad y asistencia social. Mismos que responden a indicadores y variables del modelo de Golovanesky (2007) adaptada para el caso de familias jornaleras migrantes: a. Vivienda: dado que las familias jornaleras migrantes se asientan temporalmente en albergues que han dispuesto las asociaciones de cañeros, el dato más importante, en la medición cuantitativa fue determinar el grado de hacinamiento que existe durante su estancia laboral, debido a que desencadena otro tipo de problemáticas al interior de los albergues.

b. Capital humano: que refiere a aquellas habilidades y conocimientos que pueden traducirse y emplearse en formas y estilos de vida (Sen, 1998), se concibió necesario explorar los oficios o conocimiento empírico especializado que puedan tener las mujeres migrantes.

c. Empleo: aunque la mayoría de las familias jornaleras migrantes, los hombres en calidad de padres de familias o hijos, se insertan a la actividad del corte de caña, de forma reciente se ha tenido registro, no formal, que algunas mujeres se emplean en actividades agrícolas, principalmente en invernaderos, por lo que se consideró pertinente explorar la ocupación y su cualificación.

d. Protección social: aunque se parte de forma previa de concebir que quienes se insertan a la actividad de corte de caña, tienen acceso a servicios de salud, no se sabe con certeza, si es una realidad para todos los trabajadores, por el tipo de empleo, que no siempre es formal, por lo que fue imperioso explorar el servicio de salud, al mismo tiempo, de conocer la recepción de ayuda gubernamental o de Asociaciones Civiles.

e. Rasgos demográficos: este indicador fue para definir los elementos fundamentales del perfil de la población, es decir, sexo, edad, escolaridad y lugar de nacimiento.

\subsection{Población y muestra}

Bajo la lógica de generar datos sociodemográficos de la población, se concibió una especie de censo ya que la naturaleza de su migración pendular -que implica una estancia temporal de aproximadamente seis meses en los albergues del municipio de Cuauhtémoc en el estado de Colima- no permite tener un registro exacto de la población que año con año llega para el periodo de zafra (Secretaría de Gobernación, 2018). En ese sentido, la intención del cuestionario, fue por un lado, generar datos para la administración de los albergues, pues es información que les permite dirigir algunas acciones de intervención durante el periodo de zafra, y por el otro, generar información para cumplir con el objetivo de estudio.

Para aplicar el cuestionario fue necesario identificar a un o una informante por cada habitación de los albergues, que pudiera reportar los datos de todas las personas con las que 
estuviera viviendo durante ese periodo, y en su caso, aplicar al total de las personas solas.

Para controlar y registrar la aplicación de la encuesta, se realizó un registro, tipo croquis, de cada albergue, para señalar las habitaciones encuestadas y que facilitara el regreso a aquellas en las que no se hubiesen aplicado.

\subsection{Criterios de inclusión de las personas informantes}

Como informantes se buscó a mujeres que estuvieran a cargo de la familia por cada habitación del albergue, o personas mayores de 15 años, que pudieran otorgar información del total de las personas con las que comparte el espacio; mientras que, para las personas solas, que fueran jornaleros migrantes que dieran sus datos generales, o mostrar sus documentos oficiales para el registro.

\section{VULNERABILIDAD SOCIAL DE MUJERES MIGRANTES DE FAMILIAS JORNALERAS}

Los resultados obtenidos se presentan siguiendo el modelo de Golovanevsky (2007), para evidenciar los niveles macro y micro objetivos que configuran la vulnerabilidad social a la que están expuestas las mujeres migrantes.

\subsection{Recursos que tienen las mujeres migrantes de familias jornaleras}

Este primer elemento, alude a rasgos demográficos de la población, se encuestaron a 350 personas que se encontraban ubicadas en los 6 albergues: Cóbano (84), Cuauhtémoc (19), Plaza de Toros (89), Simón Bolívar (54), Trapiche (86) y Zedillo (18), todos localizados en el municipio de Cuauhtémoc, en Colima, México, durante el periodo de noviembre de 2018 a abril de 2019 (ver gráfica 1).

Del total de personas encuestadas el $67 \%$ fueron mujeres, en calidad de madres, esposas o hijas que participaron en la encuesta y en quienes se centra los resultados de este análisis preliminar. Una característica importante que contribuye a la configuración de las dinámicas de las mujeres en su vida cotidiana y, que influye en la construcción de sus recursos personales, familiares y comunitarios, es la vivienda. En ese sentido, resultó importante explorar el número de integrantes en la vivienda temporal, en las que están localizadas las mujeres referidas.

Como se observa en la gráfica 2 y de acuerdo con el índice de calidad global de la vivienda ${ }^{3}$, los albergues para jor-
Gráfica 1. Personas encuestadas en los albergues

Mujeres Hombres

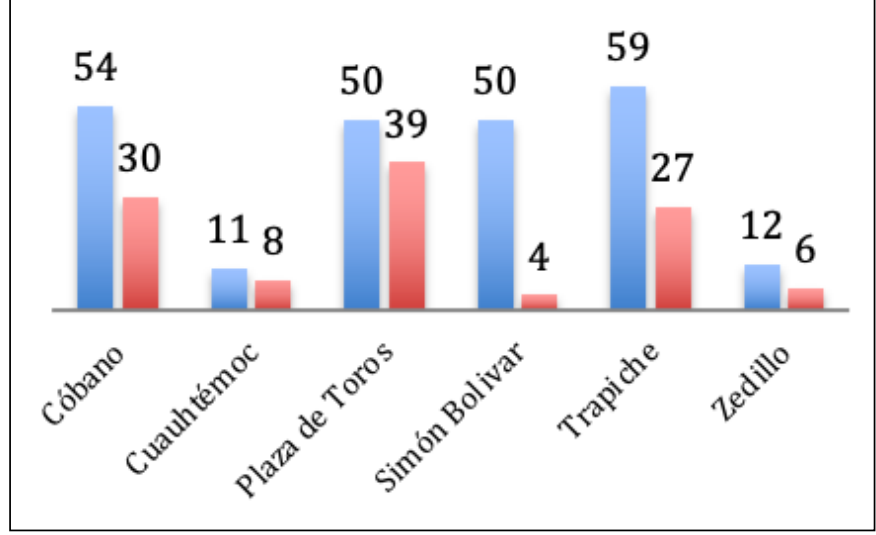

Fuente: elaboración propia (2019)

GráfICA 2. NúMERO de PERSONAS POR VIVIENDA

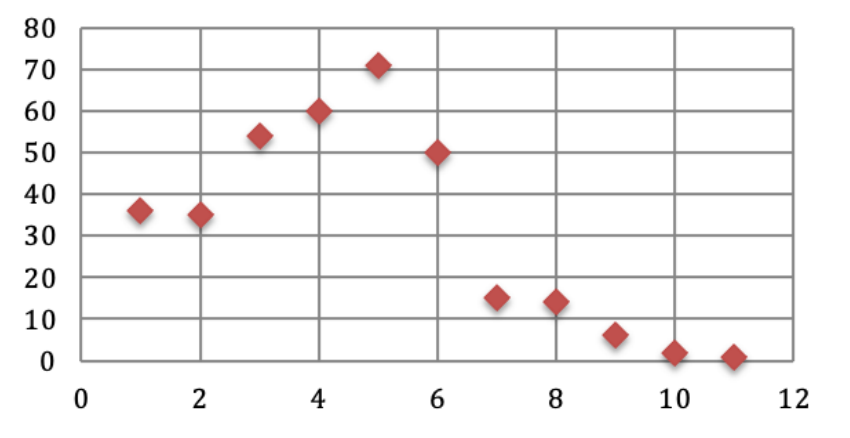

Fuente: elaboración propia (2019)

naleros presentan un hacinamiento crítico, ya que al interior de la vivienda no presentan divisiones; se identificó que 71 viviendas temporales albergan a 5 personas, seguido de 60 viviendas con 4 personas y 54 con 3 personas. La situación de hacinamiento repercute en la privacidad que, sin duda, desencadena otro tipo de problemas de cotidianos para las mujeres migrantes que radican temporalmente ahí.

La mayoría (83\%) de las mujeres migrantes en los albergues nacieron en municipios del estado de Guerrero, mientras que el $7 \%$ nació en Colima y el 5\% en Jalisco (ver gráfica 3). La edad de las mujeres, como se muestra en la gráfica 5 , aquellas que nacieron en Colima o en Jalisco, fue durante la estancia temporal de su familia de origen en dichos estados.

El estado de Guerrero, en el contexto nacional, representa el sexto estado con mayor número de personas, en edad de 5 años y más, que hablan alguna lengua indígena (Instituto Nacional de Estadítica y Geografía, 2010), situación que se

3 https://www.redatam.org/redchl/mds/casen/WebHelp/informaci $\mathrm{n}$ casen/conceptos $\mathrm{y}$ definiciones/vivienda/indice calidad global de la vivienda.htm 


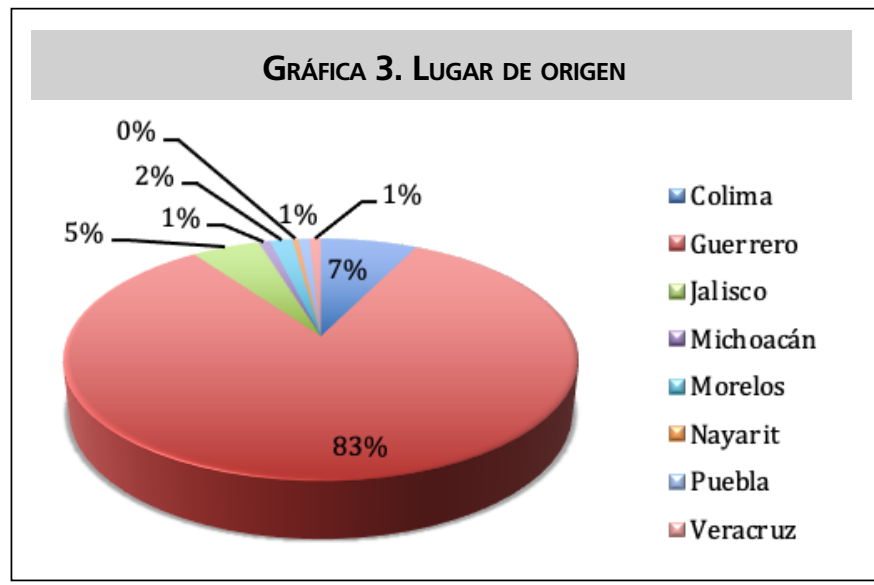

Fuente: elaboración propia (2019)

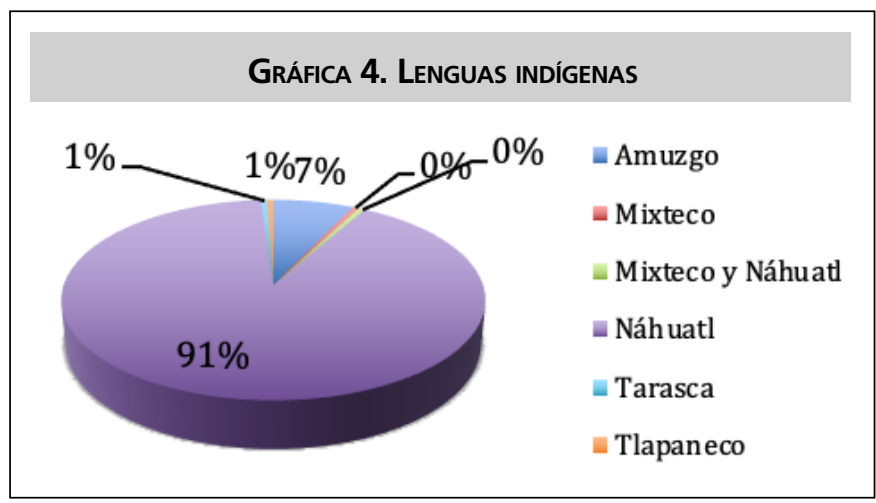

Fuente: elaboración propia (2019)

ve reflejada con la población en los albergues, ya que el $91 \%$ de las mujeres migrantes encuestadas reportó que habla Náhuatl, seguido de Amuzgo 7\% y Tarasco y Tlapaneco en igual proporción, con el $1 \%$ (ver gráfica 4 ).

Otra característica del lugar de origen y, que influyen en la limitación/acceso a los recursos que pueden tener las mujeres guerrerenses, se encuentra la situación de pobreza, que se ha definido como:

Un fenómeno multidimensional que incluye sobre los recursos, la carencia a la educación y de desarrollo de destrezas, la deficiente salud, la desnutrición, la falta de vivienda, el acceso limitado al agua, la violencia y el crimen, la ausencia de libertad política y de expresión (Silva, 2016, p. 48).

La situación de pobreza en el estado de Guerrero, se reportó de la siguiente manera, el $66.5 \%$ de la población guerrerense vive en situación de pobreza y un $26.8 \%$ se encuentra en pobreza extrema (Consejo Nacional de Evaluación de la Política de Desarrollo Social, 2019). Esta situación de su lugar de origen ha repercutido en la pobreza generacional y en el estilo de la migración temporal para obtener empleo agrícola.

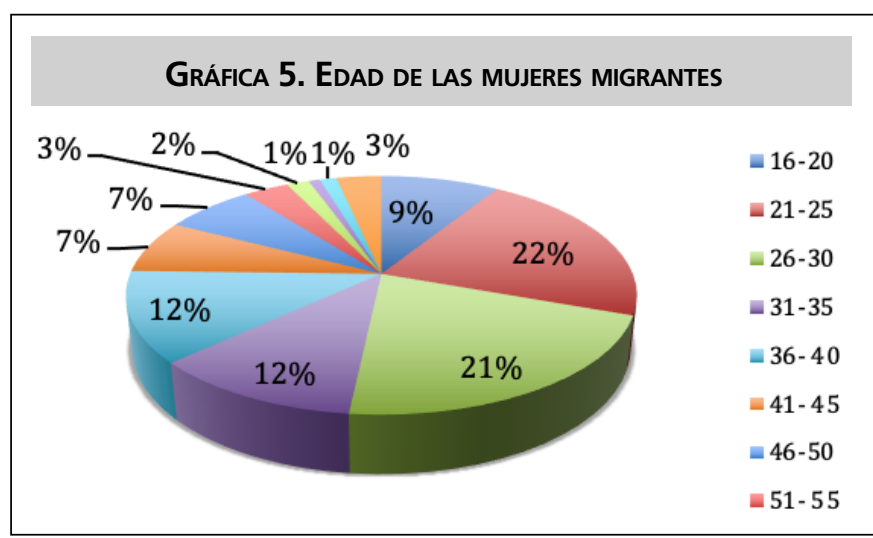

Fuente: elaboración propia (2019)

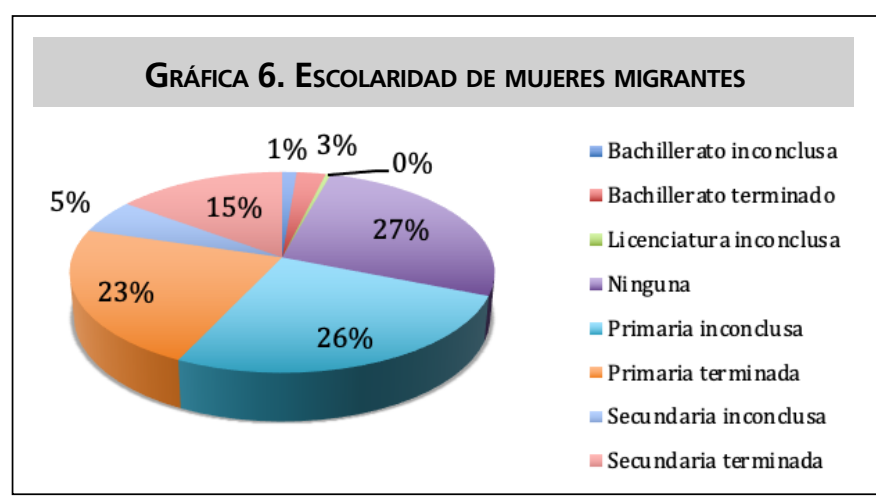

Fuente: elaboración propia (2019)

En el sentido de la imbricación de sistemas de discriminación y/o de exclusión, el lugar de origen y la lengua indígena, son condicionantes que inciden en la limitación a la infraestructura de servicios, configurando así la situación de vulnerabilidad social de las mujeres migrantes, debido a que la "población indígena reside en entornos de mayor dispersión poblacional y menor infraestructura de servicios que, en conjunto, se traducen en condiciones de vida con mayor precariedad" (Consejo Nacional de Evaluación de la Política de Desarrollo Social, 2014, p. 41).

Por otro lado, la edad de las mujeres migrantes que está representada en la gráfica 5 , indica que un $22 \%$ pertenecen al rango de 21 a 25 años, seguido del grupo de 26 a 30 años (21\%) y en igualdad de porcentaje, los grupos de 31 a 35 años y de 36 a 40 años (12\%).

La escolaridad de las mujeres migrantes encuestadas evidencia que el 27\% reportó Ninguna Escolaridad, seguido de Primaria Inconclusa con 26\%, mientras que el $23 \%$ tiene Primaria Terminada (ver gráfica 6), haciendo referencia a un rezago educativo, debido a la edad que tienen las mujeres encuestadas (ver gráfica 5).

El rezago educativo genera condiciones de vulnerabilidad social en las mujeres migrantes debido a que limita el desarrollo 
de su capital humano, ya que "la educación hace que la persona sea más eficiente en la producción de bienes, es claro que hay un mejoramiento del capital humano" (Sen, 1998, p. 69). Es decir, existe una relación directa del capital humano con las posibilidades de producción que tienen las mujeres migrantes.

\subsection{Estructura de oportunidades a la que tienen acceso las mujeres migrantes de familias jornaleras}

La estructura de oportunidades como elemento de la vulnerabilidad social, como el tipo de empleo, la protección social derivada del contrato laboral y la intervención social del Estado a través de los programas sociales.

Se identificó que las mujeres estudiadas en su mayoría $(56 \%)$ reportó ser ama de casa, es decir, se dedican exclusivamente a las labores cotidianas dentro del albergue, mientras que un 37\% manifestó ser jornalera principalmente en agro negocios (invernaderos) y un 6\% tener una doble jornada, pues se reconocen como amas de casa y auto-empleadas y/o jornaleras (ver gráfica 7).

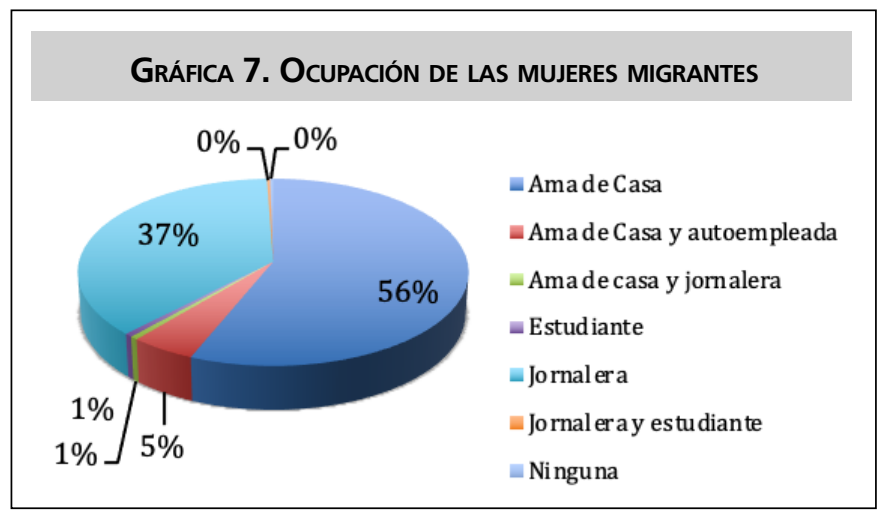

Fuente: elaboración propia (2019)

Las mujeres que desempeñan una actividad remunerada $(43 \%)$, no se reconocen como proveedoras de su familia, situación que determina la toma de decisiones al interior de la dinámica de sus hogares. Las informantes indicaron que el principal proveedor, son los jornaleros (95\%) que llegan a los albergues para incorporarse al corte de caña, como se observa en la gráfica 8 .

Otro elemento de la estructura de oportunidades es el acceso a los servicios de salud, la mayoría de las mujeres migrantes, es decir el 58\% refirieron que tienen acceso al Instituto Mexicano del Seguro Social (IMSS), seguido de un $23 \%$ a servicios del Centro de Salud y el 17\% está inscrita al Seguro Popular ${ }^{4}$ (ver gráfica 9).

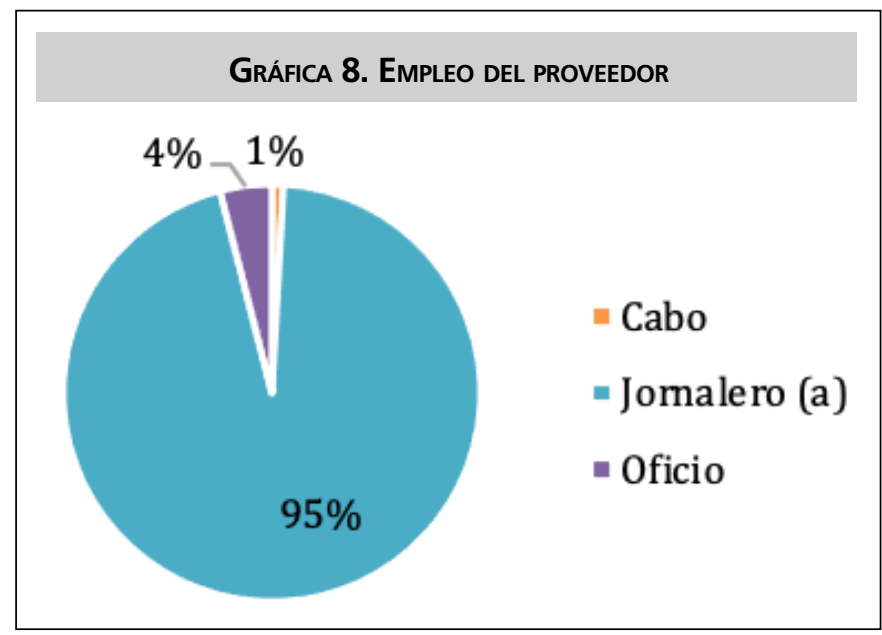

Fuente: elaboración propia (2019)

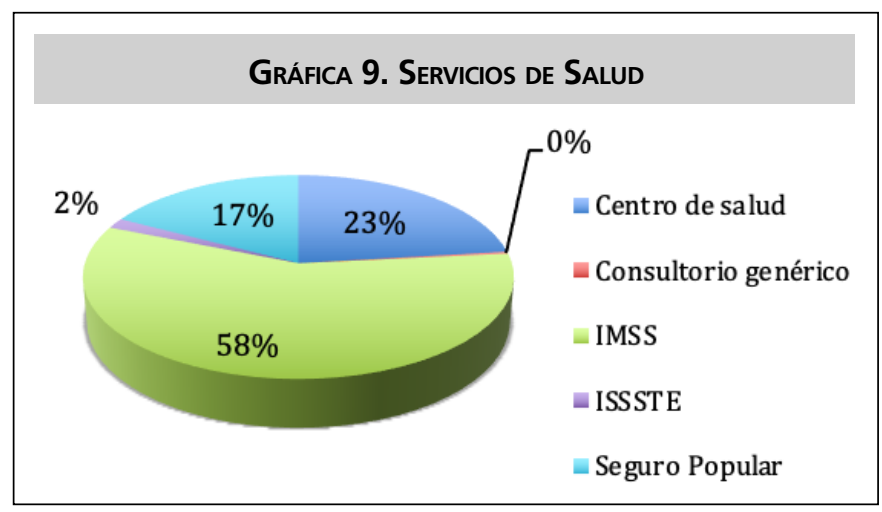

Fuente: elaboración propia (2019)

El acceso que tienen las mujeres migrantes a los servicios de salud está directamente relacionado con el empleo que tiene el principal proveedor de la familia, servicio temporal, que dura aproximadamente seis meses, mientras que la otra mitad de año está incierto el acceso que tienen a servicios de salud.

\subsection{Configuración de relaciones sociales de las mujeres migrantes}

Este eje hace alusión principalmente al capital social y se refiere a la forma en la que las mujeres migrantes tejen y configuran sus relaciones sociales y su organización social, familiar y amical que puede convertirse en una red de apoyo. En el sentido de que la migración pendular alude "al tránsito de su lugar de origen a las zonas de trabajo y de regreso" (Secretaría de Desarrollo Social, 2010, p. 15).

El $69 \%$ de las mujeres migrantes presenta un desplazamiento pendular, pues señalaron que su estancia en el albergue es durante el periodo de zafra, como se puede ilustra en

4 Programa de salud que desapareció en el 2020, pues se creó el Instituto de Salud para el Bienestar (Insabi) 


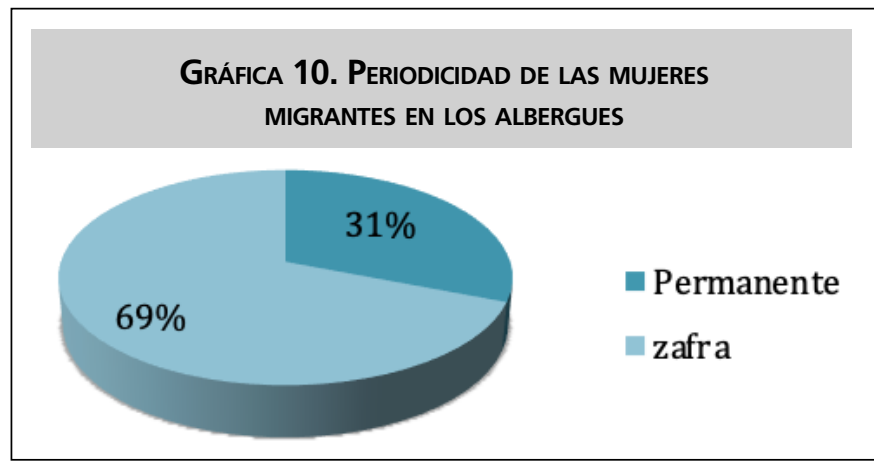

Fuente: elaboración propia (2019)

la gráfica 10, indicando los patrones circulares del péndulo alusivo a su tipo de migración.

Esta condición de desplazamiento de la migración pendular de las mujeres migrantes y sus familias, limita el desarrollo de su capital social, concepto que indica el "conjunto de recursos reales y potenciales vinculados a la posesión de una red durable de relaciones más o menos institucionales que procura beneficios de reconocimiento mutuo" (Bourdieu, 1986 citado en Lozares, López, Verd, Martí, y Molina, 2011, p. 2).

Debido a ello, la movilidad geográfica de las mujeres migrantes genera condiciones que abonan a la vulnerabilidad social a la que están expuestas, pues no desarrollan su red de relaciones que pudiese incidir favorablemente durante su estancia temporal en los albergues. Limitando así, su posicionamiento ante los administradores, los contratistas y con la sociedad en la que se asientan temporalmente, pues para poder desarrollar el capital social es necesario que "la estructura de relaciones/redes que se configuran en instituciones, asociaciones a partir de una base sustantiva constituida por la confianza, la reciprocidad y las normas compartidas" (Lozares et al., 2011, p. 3), que les es imposible desarrollar y afianzar por la temporalidad de su estancia.

\section{CONSIDERACIONES FINALES}

El alcance del estudio fue exploratorio para describir la vulnerabilidad social a la que están expuestas las mujeres migrantes de las familias jornaleras localizadas en los seis albergues del municipio de Cuauhtémoc en el estado de Colima, que representó al $67 \%$ de la población asentada durante el periodo de zafra (2018-2019).

El acercamiento a elementos macro y micro objetivos de la vulnerabilidad social a la que están expuestas las mujeres migrantes en los albergues, lleva a considerar que existen factores que responden a la interseccionalidad, es decir, "la expresión de un sistema complejo de estructuras de opresión que son múltiples y simultáneas" (Cubillos, 2015, p. 122) y que es una la realidad para las mujeres estudiadas.

La interseccionalidad está en concordancia, con el paradigma crítico, que refiere a que el análisis social no puede considerar solo a la población, sino que es necesario incorporar el contexto histórico, que refiere a que las mujeres migrantes pertenecen a familias que han configurado un estilo de vida jornalera agrícola; aunado al contexto geográfico, el lugar de origen de las mujeres migrantes ha condicionado el acceso o la limitación las oportunidades que tienen.

Los resultados refirieron que, en los albergues del municipio de Cuauhtémoc, en Colima, en su mayoría se encuentran mujeres guerrerenses (83\%), provenientes de un estado pobre (66\% en pobreza y un $26.8 \%$ pobreza extrema), de origen indígena, que son hablantes de Náhuatl (91\%), que en suma, son elementos que configuran su vulnerabilidad social. Aunado a que su "identidad [...] se configura en el marco de un sistema capitalista y patriarcal, que divide a la población con base en la raza y género" (Molina, 2020, p. 110).

Asimismo, presentan un rezago educativo, ya que el grueso de la población está en el rango de 21 a 40 años representando el $66 \%$ del total y reportando que el $76 \%$ se encuentran en los niveles educativos de Ninguna, Primaria Inconclusa y Primaria Terminada. En su ocupación, las mujeres migrantes en un $56 \%$ son amas de casa y un $6 \%$, tiene una doble jornada, como amas de casa y auto empleadas $(6 \%)$, porcentajes importantes que no son considerados para el diseño de la política social. Tal es el caso, de los programas de la Secretaría del Trabajo y Previsión Social, que dirigen sus programas a la movilidad laboral para el sector agrícola, certificación de competencias laborales, erradicación del Trabajo Infantil Agrícola, Vinculación interinstitucional, Inspecciones en campo agrícolas y talleres de capacitación sobre derechos laborales de las mujeres jornaleras agrícolas (Red Nacional de Jornaleros y Jornaleras Agrícolas, 2019). Estos talleres están dirigidos al $37 \%$ de las mujeres estudiadas. Esto no significa, que las mujeres jornaleras migrantes no tengan una situación de vulnerabilidad social, pero se desea resaltar al resto que, en suma, representa a la mayoría en el caso estudiado.

Los factores de vulnerabilidad social y la diferencia que existe, entre ser mujer migrante o ser mujer jornalera migrante, pueden situarse como parte de los estudios sobre los "feminismos periféricos postcoloniales o del tercer nivel [que] evidencian la(s) realidad(es) de la diversidad de mujeres, de sus agencias, y sus capacidades de actuación" (Duarte, 2013, p. 161), para complejizar las diferencias que existen en las mujeres de las familias jornaleras migrantes. 
Los resultados preliminares obtenidos evidenciaron los pocos recursos que han podido desarrollar las mujeres migrantes, en cuanto a la estructura objetiva se refiere, debido al hacinamiento en la que residen temporalmente en el lugar de destino, así como una limitación al desarrollo de su capital humano, por las condiciones de su lugar de origen y la actividad laboral de sus familias, que influye en su acceso a la educación básica.

La migración temporal en su desplazamiento pendular ha condicionado la forma en la que las mujeres migrantes establecen sus relaciones sociales, complejizando su posicionamiento y su red de relaciones en los lugares en los que se asientan temporalmente, que restringen el desarrollo de su red social de apoyo.

El supuesto de investigación es afirmativo, pues la mayoría de las mujeres migrantes en los albergues que no se incorpora a una actividad laboral ni asisten a una institución educativa, al momento, no se les ha visibilizado, por lo tanto, suman mayores elementos a su vulnerabilidad social.

Estas discusiones se hacen para concebir que desde el trabajo social crítico se busca comprender "al mundo y al ser en las múltiples dimensiones que lo condicionan y a la vez posibilitan la transformación de sus pensamientos y de su actuación para la construcción de un mundo más justo y solidario" (Mancinas, Zúñiga, y Arroyo, 2017, p. 35). Reconociendo que "los desplazamientos de lugar, así como traen nuevos modelos, traen rechazo y discriminaciones de la gente de la zona a la que llega, ya sea de su propia comunidad como de la gente que les considera como una carga que deriva en un constante estrés por adaptarse o no a las costumbres de la comunidad donde estará de paso" (Molina, 2020, p. 111).

Los resultados evidenciados, dan la pauta para hacer una serie de discusiones y toma de postura que son necesarias para el Trabajo Social, pues puede marcar una línea base, de la cual parte para transitar a estudios mixtos y multidisciplinares que den los elementos para diseñar estrategias de intervención más dirigidas.

En ese mismo tenor, el Trabajo Social crítico busca "revisar [...] la manera de participar, de intervenir en el contexto particular de cada territorio, adecuándonos a las características que lo diferencian de otro/s, considerando la/s pobreza/s, cuantitativas/ cualitativas, carencias, precarización, indigencia, desempleo, exclusión, des-ciudadanización y la disponibilidad de Políticas Públicas/Sociales que den respaldo" (Pérez y Oliva, 2014, p. 2).

También, desde la profesión, reconocer que la discriminación múltiple a la que están expuestos algunos grupos vulnerables, como resultan ser las mujeres migrantes estudiadas, quienes en su ocupación de amas de casa, y algunas en su doble jornada laboral, no se les ha reconocido el valor de su trabajo cotidiano para la dinámica de los jornaleros migrantes y de sus propias familias dentro del fenómeno migratorio agrícola.

La evolución del Trabajo Social, en cuanto a su práctica y teoría "la constante adaptación a la realidad social en la que [... se encuentran] inmersos como profesionales y disciplina" (Juan, 2009, p. 56), permite hacer análisis integrales que contribuyan a la transformación, para buscar la inclusión social de aquellos grupos más vulnerables. En consecuencia, considerar que "los factores de exclusión social se retroalimentan dentro de un entramado multicausal y complejo que requiere de respuestas profesionales integrales" (Juan, 2009, p. 55), como ha sido la propuesta exploratoria aplicada para describir el espacio temporal de las mujeres desde la estrategia exploratoria de análisis sobre la vulnerabilidad social desde la interseccionalidad que se presentó.

Todo ello, para velar por los principios que rigen al Trabajo Social, buscar "la justicia social, los derechos humanos, la responsabilidad colectiva y el respeto a la diversidad" (Federación Internacional de Trabajadores Sociales, 2014, párr. 1), a través de entender el presente y reconociendo la complejidad del pasado, la contextualización geográfica y cultural a la que pertenecen las mujeres migrantes; además, asumir que la migración es un derecho humano y no un problema en sí mismo, y, que las mujeres referidas están delimitadas y contextualizadas dentro de estructuras que configuran su realidad social (lugar de nacimiento, etnicidad, sexo, educación, estado civil, núcleo familiar, estado civil, ocupación, etc.).

\section{REFERENCIAS BIBLIOGRÁFICAS}

Brah, A. (2013). Pensando en y a través de la interseccionalidad. En M. Zapata, S. García, y J. Chan (Eds.), La interseccionalidad en debate. Actas del Congreso Internacional. Indicadores Interseccinales y Medidas de Inclusión Social en Instituciones de Educación Superior. Berlín: Miseal. 14- 20 . Disponible en: https://www.upla.cl/inclusion/wp-content/uploads/2015/04/Interseccionalidadendebate misealweb-1.pdf

Consejo Nacional de Evaluación de la Política de Desarrollo Social (2014). La pobreza en la población indígena de México, 2012: https://www.coneval.org.mx/Informes/ Coordinacion/INFORMES Y PUBLICACIONES PDF/POBREZA POBLACION INDIGENA 2012.pdf (13 May. 2018)

Consejo Nacional de Evaluación de la Política de Desarrollo Social (2019). Diez años de medición de pobreza multidimensional en México: avances y desafíos en política social. México: https://www.coneval.org.mx/Medicion/ 
MP/Documents/Pobreza 18/Pobreza_2018 CONEVAL. pdf (14 Agos. 2020)

Cubillos, J. (2015). La importancia de la interseccionalidad para la investigación feminista. Oxímora Rvista Internacional de Ética y Política, 7, 119- 137. Disponible en: http:// revistes.ub.edu/index.php/oximora/article/view/14502

Duarte, C. M. (2013). La interseccionalidad en las políticas migratorias de la Comunidad de Madrid. Punto Género, 167-194. Disponible en: https://revistapuntogenero.uchile.cl/index.php/RPG/article/view/30274

Federación Internacional de Trabajadores Sociales (2014). Definición Mundial del Trabajo Social: https://www.ifsw. org/what-is-social-work/global-definition-of-social-work/ definicion-global-del-trabajo-social/(13 Ene. 2017)

Golovanevsky, L. (2007). Vulnerabilidad Social: una propuesta para su Medición en Argentina. Revista de Economía y Estadística, 45, 53-94. Disponible en: http://revistas.unc. edu.ar/index.php/REyE/article/view/3840

Grupo Sinergia, C. S. (2011). Diagnóstico Regional y Comunitario. Zona de Influencia del Ingenio Quesería. Beta San Miguel S.A de C.V. Cuauhtémoc.

Instituto Nacional de Estadística y Geografía (2010). Temas: lenguas: https://www.inegi.org.mx/temas/lengua//8 Jul. 2019)

Juan, E. M. (2009). Vulnerabilidad, exclusión social, desigualdad y ciudadanía. El Trabajo social ante situaciones de desigualdades estructurales. Documentos de Trabajo Social, 45, 49-57. Disponible en: https://dialnet.unirioja.es/ servlet/articulo? codigo $=3288168$

La Barbera, M. C. (2016). Interseccionalidad, un "concepto viajero": orígenes, desarrollo e implementación en la Unión Europea. Interdisciplina, 4, 105-122. Disponible en: http://revistas.unam.mx/index.php/inter/article/ view/54971

Lozares, C., López, P., Verd, J., Martí, J., y Molina, J. L. (2011). Cohesión, Vinculación e Integración sociales en el marco del Capital Social. REDES- Revista hispana para el análisis de rede sociales. 20, 1-28. Disponible en: http://revista-redes.rediris.es/pdf-vol20/vol20 1.pdf

Mancinas, S. E. (2017). Paradigma Crítico y Trabajo Social. En S. E. Mancinas, M. Zúñiga, C. Arroyo, L. M. Rodríguez, y B. M. Tamez (Eds.), Teorías y Modelos de intervención en Trabajo Social. Fundamentos básicos y crítca (pp. 153192). México: Res Pública.

Mancinas, S. E., Zúñiga, M., y Arroyo, C. (2017). Paradigmas de las Ciencias Sociales, Trabajo Social e Intervención. En Teorías y Modelos de Intervención Social. Fundamentos Básicos y Critica (pp. 23- 55). Ciudad de México: Res Pública.
Molina, N. E. (2020). Ser mujer indígena, náhuatl, casada, migrante, sin trabajo remunerado: una realidad en los albergues para jornaleros agrícolas en Colima, México. Prospectiva. Revista de Trabajo Social e Intervención Social, 30, 91-116. Disponible en: https://revistaprospectiva. univalle.edu.co/index.php/prospectiva/article/view/8547

Olmos, E. S. (2016). Diagnóstico Social. Universidad de Colima. Colima.

Pérez, M. d. V., y Oliva, M. A. (2014). Trabajo social. "pobreza/s" e intervención en el contexto latinoamericano. La diversidad como desafío. Catamarca. Margen, 72, 1- 9. Disponible en: http://www.margen.org/suscri/margen72/ fuentes.pdf

Pimienta, R., \& Vera, M. (2015). Migración interna en México. Datos de la muestra censal de 2010: http://ri.uaemex. mx/handle/20.500.11799/40514 (8 Abr. 2018)

Programa de las Naciones Unidas para el Desarrollo (2015). Objetivos de Desarrollo Sostenible: http://www.undp.org/ content/undp/es/home/sustainable-development-goals. html(03 Nov. 2020)

Red Nacional de Jornaleros y Jornaleras Agrícolas. (2019). Violación de derechos de las y los jornaleros agrícolas en México: http://cecig.org.mx/wp-content/uploads/2019/03/ INFORME_RNJJA_2019.pdf (03 Nov. 2020)

Ritzer, G. (2002). Teoría sociológica moderna. España: Mc Graw Hill.

Ruíz, N. (2012). La definición y medición de la vulnerabilidad social. Un enfoque normativo. Investigaciones Geográficas, 63-74. Disponible en: http://www.scielo.org.mx/pdf/ igeo/n77/n77a6.pdf

Secretaría de Desarrollo Social. (2010). Diagnóstico del Programa de Atención a Jornaleros Agrícolas: http:// www.inapam.gob.mx/work/models/SEDESOL/Resource/1778/3/images/Diagnostico PAJA.pdf (03 Abr. 2017)

Secretaría de Gobernación. (2018). Ficha temática. Personas jornaleras agrícolas: http://www.conapred.org.mx/userfiles/files/FichaPjornalera.pdf (03 Nov. 2020)

Sen, A. (1998). Capital humano y capacidad humana. Cuadernos de economía, 67-72. Disponible en: https://dialnet.unirioja.es/servlet/articulo?codigo $=4934956$

Silva Arcienega, R. (2016). Dimensiones psicosociales de la pobreza percepción de una realidad recuperada. Ciudad de México: UNAM.

Viveros Vigoya, M. (2016). La interseccionalidad: una aproximación situada a la dominación. Debate feminista, 56, 117. Disponible en: https://www.sciencedirect.com/science/ article/pii/S0188947816300603 